\section{INJURIES ARE NEGATIVELY ASSOCIATED WITH TEAM PERFORMANCE IN PROFESSIONAL CRICKET}

1,2Luke Goggins, ${ }^{2}$ Nicholas Peirce, ${ }^{2}$ Steve Griffin, ${ }^{2}$ Ben Langley, ${ }^{1}$ Keith Stokes, ${ }^{1}$ Carly McKay, ${ }^{1}$ Sean Williams. 'University of Bath, Bath, UK; ${ }^{2}$ England and Wales Cricket Board, Loughborough, UK

\subsection{6/bjsports-2021-IOC.38}

Background A negative association between injuries and team success has been demonstrated across a number of sports, but the nature of this association in professional cricket teams is currently unclear.

Objective To examine the relationship between injuries and team success in professional cricket.

Design A retrospective analysis of all match time-loss injuries and County Championship point tallies for nine seasons (from 2010 - 2018 inclusive).

Setting Eighteen First-Class County Cricket (FCCC) cricket teams in England and Wales.

Patients (or Participants) Professional male senior first-team cricketers registered to one of the eighteen clubs (mean $\mathrm{n}=$ 507 players/season).

Interventions (or Assessment of Risk Factors) Two injury measures were assessed for within-team (linear mixed model) and between-team (correlation) effects; match time-loss injury incidence and burden, to account for both the frequency and severity (days lost from competition and practice) of injuries.

Main Outcome Measurements County Championship league points tally was used as the measure of team success.

Results A moderate negative correlation was found between injury burden and team performance $(\mathrm{r}=-0.36$; 90\% CI: 0.7 to 0.1 ; likely negative). A reduction in match injury incidence of two match time-loss injuries per 1,000 days of play within a team (90\% CI 1.4 to 3.0 ; possibly negative), or a reduction in match injury burden of 74 days per 1,000 days of play (90\% CI 50.2 to 109.0 ; possibly negative) in any given season was associated with the smallest worthwhile change in County Championship points ( +13 points) for Division 1, but not for Division 2.

Conclusions Moderate reductions in injury burden potentially have worthwhile effects on performance for a domestic cricket team in the County Championship Division 1.

\section{RISK FACTORS FOR DOMINANT SHOULDER INJURY IN ELITE FEMALE AUSTRALIAN CRICKET PLAYERS: A PROSPECTIVE STUDY}

1,2,3 Myles Murphy, ${ }^{4,5}$ Paola Chivers, ${ }^{6}$ Kate Mahony, ${ }^{7}$ Andrea Mosler. ${ }^{1}$ School of Physiotherapy, The University of Notre Dame Australia, Fremantle, Australia; ${ }^{2}$ SportsMed Subiaco, St John of God Health Care, Subiaco, Australia; ${ }^{3}$ Australian Centre for Research into Injury in Sport and its Prevention, Edith Cowan University, Joondalup, Australia; ${ }^{4}$ Institute for Health Research, The University of Notre Dame Australia, Fremantle, Australia; ${ }^{5}$ Exercise Medicine Research Institute and School of Medical and Health Sciences, Edith Cowan University, Joondalup, Australia; ${ }^{6}$ Performance Health, New South Wales Institute of Sport, Sydney Olympic Park, Australia; ' La Trobe Sports and Exercise Medicine Research Centre, La Trobe University, Bundoora, Australia

\subsection{6/bjsports-2021-IOC.39}

Background In elite Australian cricket, shoulder injuries account for $11 \%$ of all injuries and $5.8 \%$ of all time-loss injuries in female players. However, even with over 400,000 females participating in cricket within Australia there are no studies exploring the risk factors for shoulder injury.
Objective Examine the risk factors for dominant shoulder injury in elite female cricketers during the 2017-2018 season. Design Prospective cohort study.

Setting Australian national cricket league.

Participants A total of 115 elite, female cricketers were included with a mean (SD) age of $26.0(4.4)$ years. 39 players had missing data for some pre-season risk factors; however, all players were monitored for injury throughout the entirety of the 2017-2018 season.

Assessment of Risk Factors Univariate and multivariate logistic regression determined the relationship between aerobic fitness and musculoskeletal screening tests with incidence of dominant shoulder injury.

Main Outcome Measurements Pre-season aerobic fitness, musculoskeletal screening tests (shoulder range of motion, shoulder strength, hip strength, scapula dyskinesis, hypermobility and combined elevation) and dominant shoulder injury requirirng modification of throwing were recorded.

Results Fourteen players developed dominant shoulder injuries however as two resulted from trauma these were excluded so 12 injuries proceeded to analysis. Univariate analysis revealed shoulder internal rotation: external rotation (IR:ER) strength ratio $(\mathrm{OR}=1.84, \mathrm{p}=0.01)$, back foot hip abduction strength $(\mathrm{OR}=0.973, \mathrm{p}=0.049)$ and back foot hip adduction: abduction strength ratio $(\mathrm{OR}=1.44, \mathrm{p}=0.047)$ were significantly associated with injury. Only shoulder IR:ER strength ratio remained significant $(p=0.016)$ in the multivariate logistic regression model with a $79 \%$ increased risk of shoulder injury for every 0.1 ratio increase.

Conclusions This study identified that within elite female cricketers, a shoulder IR:ER strength ratio of greater than 1.00 is the strongest risk factor for developing shoulder injury. Therefore, injury risk reduction programs in elite female cricketers which focus on keeping the shoulder IR:ER strength ratio closer to $1: 1$ may assist to minimise shoulder injury burden.

\section{CHANGE OF DIRECTION BIOMECHANICS AND THE RISK FOR NON-CONTACT KNEE INJURIES IN YOUTH BASKETBALL AND FLOORBALL PLAYERS}

${ }^{1}$ Mari Leppänen, ${ }^{1}$ Jari Parkkari, ${ }^{2}$ Tommi Vasankari, ${ }^{3}$ Sami Äyrämö, ${ }^{4}$ Tron Krosshaug, ${ }^{1,5}$ Pekka Kannus, ${ }^{1,6,7,8}$ Kati Pasanen. 'Tampere Research Center of Sports Medicine, UKK Institute, Tampere, Finland; ${ }^{2}$ UKK Institute, Tampere, Finland; ${ }^{3}$ Faculty of Information Technology, University of Jyväskylä, Jyväskylä, Finland; ${ }^{4}$ Oslo Sports Trauma Research Center, Norwegian School of Sport Sciences, Oslo, Norway; ${ }^{5}$ Tampere University Hospital, Tampere, Finland; ${ }^{6}$ Sport Injury Prevention Research Centre, Faculty of Kinesiology, University of Calgary, Calgary, Canada; 'Alberta Children's Hospital Research Institute, University of Calgary, Calgary, Canada; ${ }^{8}$ McCaig Institute for Bone and Joint Health, University of Calgary, Calgary, Canada; ${ }^{9}$ Faculty of Sport and Health Sciences, University of Jyäskylä, Jyväskylä, Finland

\subsection{6/bjsports-2021-IOC.40}

Background Studies investigating biomechanical risk factors for knee injuries in sport specific tasks are needed.

Objective To investigate association between change of direction (COD) biomechanics and knee injury risk among youth team sports.

Design Prospective cohort study.

Setting Youth basketball and floorball.

Participants 258 female and male players (aged 12-21).

Assessment of Risk Factors COD biomechanics were analysed using 3D-motion analysis. The COD test consisted of a quick 